\title{
Setting a Research Agenda in Prevention of Healthcare-Associated Infections (HAIs) and Multidrug-Resistant Organisms (MDROs) Outside of Acute Care Settings
}

\author{
Charlesnika T. Evans, PhD, MPH; ${ }^{1,2}$ Robin L. Jump, MD, PhD; ${ }^{3,4}$ Sarah L. Krein, RN, PhD; ${ }^{5,6}$ Suzanne F. Bradley, MD; ${ }^{6,7}$ \\ Christopher J. Crnich, MD, PhD; ${ }^{8,9}$ Kalpana Gupta, MD; ${ }^{10,11}$ Eli N. Perencevich, MD, MS; ${ }^{12,13}$ \\ Mark W. Vander Weg, PhD; ${ }^{12,13}$ Daniel J. Morgan, $\mathrm{MD}^{14}$
}

(See introductory commentary by Livorsi et al, pages 186-188.)

Infections are among the most common complications of hospital care and a leading cause of death in the United States. ${ }^{1}$ Multidrug-resistant organisms (MDROs) cause more than 2 million infections each year, with $>1 \%$ of those resulting in death. ${ }^{2}$ Risk factors for healthcare-associated infections (HAIs) include patient characteristics (eg, cognitive impairment, spinal cord injury, or end-stage renal disease), medical treatments (eg, recent antibiotic exposure, indwelling medical devices, or dialysis), the type of care received (eg, dementia care, wound care, or rehabilitation), and the setting in which the care is delivered (eg, long-term care setting, spinal cord injury unit, or dialysis center).

Most knowledge about preventing HAIs due to MDROs comes from acute-care settings. Translating this knowledge to individuals and settings outside of hospital wards presents significant challenges. Specifically, protocols commonly used to reduce the risk of HAI and MDROs may not apply to or may be inadequate for meeting the needs of clinical staff and patient populations outside the general acutecare setting. Consequently, identification of effective practices to provide safe healthcare for patients in other settings is needed. Here, we discuss recommendations for prioritizing the research agenda for special populations and for healthcare services offered in settings beyond typical inpatient hospital units.

\section{SPECIAL POPULATIONS INCLUDED}

The workgroup prioritized selected populations in preventing and managing HAIs and MDROs. The populations included long-term care (LTC), spinal cord injury/disorder (SCI/D), rehabilitation, mental healthcare, ambulatory care, and homebased care. An 'other' category permitted group members to specify other population(s) they deemed important. In the initial round of prioritizing the research agenda for different populations, the working group ranked LTC residents first, followed by SCI/D, ambulatory care (including emergency departments and urgent care), rehabilitation, mental health, and home-based care. Two additional populations emerged, those who receive dialysis and those in hospice care. The working group further refined and grouped the populations as follows: (1) LTC including rehabilitation units, (2) SCI/D, (3) ambulatory care, (4) dialysis, (5) home-based care and hospice, and (6) mental health.

Despite disparate clinical needs, the type of care involved with LTC and SCI/D populations was similar enough to warrant crossover of evidence-based approaches for HAI and MDRO prevention. These similarities include assistance with

Affiliations: 1. Center of Innovation for Complex Chronic Healthcare (CINCCH), Department of Veterans Affairs (VA), Edward Hines Jr Veterans Affairs Hospital, Hines, Illinois; 2. Department of Preventive Medicine and Center for Health Care Studies, Feinberg School of Medicine, Northwestern University, Chicago, Illinois; 3. Geriatric Research, Education and Clinical Center and Specialty Care Center of Innovation, Louis Stokes Cleveland VA Medical Center, Cleveland, Ohio; 4. Division of Infectious Disease, Department of Medicine, School of Medicine, Case Western Reserve University, Cleveland, Ohio; 5. Center for Clinical Management Research and VA/University of Michigan Patient Safety Enhancement Program, VA Ann Arbor Healthcare System, Ann Arbor, Michigan; 6. Department of Internal Medicine, University of Michigan, Ann Arbor, Michigan; 7. Infectious Diseases Section, Geriatric Research, Education and Clinical Center, VA Ann Arbor Healthcare System, Ann Arbor, Michigan; 8. School of Medicine and Public Health, University of Wisconsin, Madison, Wisconsin; 9. William S. Middleton VA Hospital, Madison, Wisconsin; 10. Department of Medicine, Boston University, Boston, Massachusetts; 11. Section of Infectious Diseases, VA Boston Healthcare System, West Roxbury, Massachusetts; 12. Center for Comprehensive Access and Delivery Research and Evaluation, Iowa City VA Healthcare System, Iowa City, Iowa; 13. Department of Internal Medicine, Carver College of Medicine, University of Iowa, Iowa City, Iowa; 14. VA Maryland Healthcare System and University of Maryland School of Medicine, Baltimore, Maryland.

Received November 7, 2017; accepted December 2, 2017

(C) 2018 by The Society for Healthcare Epidemiology of America. All rights reserved. 0899-823X/2018/3902-0012. DOI: 10.1017/ice.2017.291 
activities of daily living, home-like environment in certain settings, long lengths of stay, and the presence of indwelling medical devices. Finally, discussion among group members during the in-person meeting identified notable variability in the level of evidence available for HAI and MDRO prevention for the 6 populations listed here. Together, these knowledge gaps informed the working group's recommendations for research; they are summarized below and are detailed in Table 1.

\section{DISCUSSION AND RECOMMENDATIONS}

Briefly, data describing the epidemiology and current practices regarding HAIs and MDROs in LTC and SCI/D populations are limited; best practices specific to these settings have not yet been established. The LTC and SCI/D populations have commonalities in identified research needs. Both MDROs and infections are common in these populations, but distinguishing colonization and infection is difficult. Criteria to make these distinctions needs to be strengthened with true urinary tract infection versus asymptomatic bacteriuria as a key area. Clinical decision-making is critical both for providing effective and timely antibiotic treatment and for preventing unnecessary treatment. In addition, research is needed to identify the most effective strategies for promoting antibiotic stewardship and judicious use of diagnostic tests. ${ }^{3}$ Data are also limited on the contribution of the healthcare environment, fomites and person-to-person contact that may contribute to transmission. ${ }^{4}$ In LTC and SCI/D settings, person-to-person contact includes both interactions between staff and patients during routine care activities as well as spontaneous social interactions among patients, and sometimes staff, such as handshakes and hugs. Patients or residents in LTC or SCI/D settings are encouraged to participate in recreational and social activities ensuring mobility and a home-like environment. These activities make the transmission of MDROs even more complex because of the potential for multiple reservoirs. ${ }^{5}$ The role of the patient, fomites, and the environment in transmission of MDROs needs to be further studied in these settings. In addition, optimal approaches are needed for implementing transmission-based precautions such as targeting those at highest risk (ie, those with wounds or devices). Management of urinary catheters as a priority research need was identified, including developing and implementing best practices for urinary catheter placement and management in a population that may be chronically catheterized. Finally, studies on the role of transitions between different healthcare settings on the spread of MDROs as well as strategies for mitigation at point of transition (eg, enhanced communication, stewardship extending beyond hospitalization) are needed.

For ambulatory care, dialysis, mental health, home-based care and hospice settings, the working group found very little evidence to inform current infection prevention and control practices. Furthermore, the working group found a paucity of data addressing even basic epidemiology in these settings.
Research targets for different ambulatory care settings (eg, hemodialysis, home care, dentistry) include assessing the prevalence and determining risk factors for HAIs and MDROs, determining adherence to the Centers for Disease Control and Prevention guidelines for infection prevention for outpatient or dialysis settings (ie, environmental cleaning), assessing barriers and facilitators to current recommended infection prevention practices in these settings, and testing new or adapted interventions to promote effective infection prevention. While studies to improve antibiotic use in the general outpatient setting have been conducted, the impacts on the reduction of MDROs, program sustainability, and large-scale implementation, as well as in select outpatient settings (ie, dialysis) have not been rigorously evaluated. ${ }^{3}$

In addition, for home-based care, there is a need to determine several factors: (1) the rates of acquisition of MDROs at home compared to healthcare settings; (2) the risk of transmission by healthcare workers when entering patients' homes, including the impact of the environment on transmission and best practice for cleaning of reusable equipment; and (3) best practice for urinary and intravascular catheter care. In the hospice environment, antibiotic use at end-of-life is an important issue. Specific needs include understanding the optimum route of administration, dosing frequency, and antibiotic classes used at the end of life, determining whether they reduce patient discomfort, and the potential contributions of hospice patients and their healthcare workers in the spread of MDROs. For mental health, other than evidence regarding influenza prevention for inpatient mental health units, few data exist on HAI and MDRO colonization or infection prevalence. This lack of data highlights a research need for epidemiology studies and point-prevalence surveys to inform recommendations for HAI and MDRO prevention in short- and long-stay psychiatric units. In addition, because of the unique restrictions in some psychiatric units, such as prohibition of alcohol-based hand gels, research is needed to test strategies to improve hand hygiene compliance when access to alcohol gel is restricted.

In conclusion, our expert group identified fundamental knowledge gaps related to prevention of HAIs and MDROs for patient populations that receive care in settings outside of acute care. In turn, these gaps suggest research priorities with the long-term goal of improving patient safety by reducing risks related to developing HAIs and acquiring MDROs. To date, most of the research to understand and prevent HAIs or MDROs has focused on acute-care populations. These gaps limit the external validity and generalizability to patients and settings that differ from most medical, surgical, or intensive care units in hospitals.

The populations and settings identified by our working group have unique patient care goals that require different infection prevention strategies from those used in most acute care settings. This is evident in Department of Veterans Affairs' innovative national program to reduce the spread of methicillin-resistant Staphylococcus aureus (MRSA). ${ }^{6}$ Although 
TABLE 1. Assessment of Current Evidence and Recommendations for Infection Prevention and Control (IPC) Research Needs to Reduce Healthcare Associated Infection (HAI) and Multidrug-Resistant Organisms (MDROs) in Selected Populations

\begin{tabular}{lll}
\hline Population & Current Evidence \\
\hline $\begin{array}{l}\text { Long-term care (LTC) } \\
\text { Spinal cord injury/ }\end{array}$ & $\begin{array}{l}\text { Epidemiological studies demonstrate clear } \\
\text { disorder (SCI/D) }\end{array}$ & $\begin{array}{l}\text { need for HAI/MDRO prevention. } \\
\text { contelines for infection prevention and } \\
\text { control (IPC) in LTC, rehabilitation, and } \\
\text { SCI/D adapted from acute care }\end{array}$
\end{tabular}

Research Needs (Specific QUERI 6-Step Model Addressed)

- Develop evidence-based criteria for distinguishing colonization from infections (to reduce antibiotic overuse $)^{1}$

- Assess the most effective strategies for promoting antibiotic stewardship and the judicious use of diagnostic tests ${ }^{1-3}$

- Evaluate the role of fomites and the environment in MDRO transmission ${ }^{2,3}$

- Identify optimal approach (eg, when to use gowns and gloves) to implementing transmission-based precautions in these settings, including among high-risk patients such as those with wounds/devices ${ }^{4}$

- Develop best practices related to urinary catheter placement, management, including how frequently chronic urinary catheters should be routinely changed ${ }^{4}$

Ambulatory care ${ }^{\mathrm{b}}$

Limited data describe risk of acquiring HAI/MDRO in these settings and current infection prevention practices in use.

- High incidence of MRSA in hemodialysis patients

- Adherence to infection prevention strategies has been suboptimal

Home-based care and hospice

Very limited information about infection control needs in these populations
Mental health (inpatient and outpatient)
- Primary infections in mental health are $S$. aureus, influenza, scabies and norovirus

- Alcohol-based hand gel placement limited in inpatient settings
- Assess prevalence and determine risk factors for HAI and $\mathrm{MDROS}^{1-3}$

- Assess adherence to current practices based on CDC guide to infection prevention for outpatient settings (ie, education and training, surveillance process activities, hand hygiene programs, environmental cleaning $)^{1-3}$

- Assess barriers and facilitators to current recommended IPC practices ${ }^{1-3}$

- Identify and test novel interventions or adapt interventions used in other settings to promote effective IPC $^{4}$

- Assess stewardship activities and to what extent ambulatory encounters contribute to the acquisition of MDROs $^{1-3}$

- Test barriers and facilitators to implementation of CDC guidelines in dialysis centers ${ }^{4}$

- Determine when and how to effectively use barrier precautions in dialysis ${ }^{4}$

- Determine how to perform antimicrobial stewardship in dialysis centers ${ }^{4}$

- Determine rates of acquisition of MDROs at home compared to healthcare settings ${ }^{1}$

- Assess the risk of transmission by healthcare workers when entering patients' homes ${ }^{1}$

- Determine best practice for cleaning of reusable medical equipment ${ }^{2-4}$

- Assess best practice for urinary and intravascular catheter care in home care ${ }^{2-4}$

- Determine whether there is any benefit for antimicrobial use in hospice or a need for stewardship ${ }^{4}$

- Determine whether there is any benefit for MDRO screening or decolonization in these settings ${ }^{4}$

- Assess the epidemiology of key infectionsStaphylococcus aureus, influenza, scabies, norovirus ${ }^{1}$

- Test strategies to improve hand hygiene compliance when access to alcohol gel are restricted. ${ }^{4}$

NOTE. CDC, Centers for Disease Control and Prevention.

${ }^{a}$ Includes rehabilitation.

${ }^{\mathrm{b}}$ Includes clinics, ambulatory surgical centers, emergency departments, and urgent care.

this was a nationwide healthcare initiative, the need to implement infection control practices, such as contact precautions, was in line with patient care goals in diverse settings, such as
LTC and SCI units. ${ }^{7-10}$ This effort highlighted the need for modified guidelines for implementation of the MRSA program in these units; a key component was sustaining a home-like 
environment while supporting patient safety. ${ }^{7-10}$ Our workgroup identified similar issues as a critical part of the research agenda for LTC and SCI populations.

For ambulatory care, dialysis, mental health, home-based care, and hospice care settings, the research needs appear to be even more basic given a general dearth of information about current infection prevention and control practices in these areas. Moreover, patients in these special populations or settings are often treated by specialists who have their own society guidelines. Even with national infection control and prevention policies or initiatives, these recommendations often do not filter down to specialty care providers. Further collaboration between internal medicine, geriatrics, nursing, and specialty care providers will help identify needs for and, ultimately, facilitate the implementation of practice changes.

Finally, additional factors that affect the complexity of special populations and their care environments, such as long lengths of stay, group therapy or activities, and multiple-bed rooms or units, can further complicate the prevention and management of HAIs and MDROs. We believe that addressing these issues as well as the questions outlined in this research agenda are essential steps toward reducing infection risk and improving patient care beyond the general acute-care setting.

\section{ACKNOWLEDGMENTS}

This work was supported in part by funding from the VA Health Services Research and Development (HSR\&D) Service's Center of Innovation for Veteran-Centered and Value-Driven Care (COIN) conference supplement for "Setting the Clinical Research Agenda for MDROs in VA" (grant no. CIN 13-412), from a Presidential Early Career Award for Scientists and Engineers (grant no. USA 12-564 to C.T.E.), from the VA Quality Enhancement Research Initiative CARRIAGE Program (grant no. IP1 HX001993-01A1 to C.T.E. and E.N.P.), from the VA HSR\&D Pilot Merit Program (grant no. PPO 16-118-1 to R.L.P.J.), and from a VA HSR\&D Research Career Scientist Award (grant no. RCS 11-222). The views expressed in this article are those of the authors and do not necessarily reflect the position or policy of the Department of Veterans Affairs or the US government. All authors report no conflicts of interest or financial disclosures relevant to this article.

Address correspondence to Charlesnika T. Evans, PhD, MPH, Edward Hines Jr VA Hospital, 5000 S. 5th Avenue (151H), Building 1, Room D302, Hines, IL 60141 (Charlesnika.evans@va.gov).

\section{REFERENCES}

1. 2013 annual hospital-acquired condition rate and estimates of cost savings and deaths averted from 2010 to 2013. Agency for Healthcare Research and Quality website. http://www.ahrq. gov/professionals/quality-patient-safety/pfp/index.html. Published October 2105. Accessed April 17, 2017.

2. Antibiotic resistance threats in the United States, 2013. Centers for Disease Control and Prevention website. http://www.cdc.gov/ drugresistance/threat-report-2013/pdf/ar-threats-2013-508.pdf. Published 2013. Accessed April 17, 2017.

3. Suda KJ, Livorsi DJ, Goto $M$, et al. Research agenda for antimicrobial stewardship in the Veterans Health Administration. Infect Control Hosp Epidemiol 2018;39:196-201.

4. Perencevich EN, Harris AD, Pfeiffer CD, et al. Establishing a research agenda for preventing transmission of multidrugresistant organisms in acute care settings in the Veterans Health Administration. Infect Control Hosp Epidemiol 2018;39: 189-195.

5. Patel PK, Mantey J, Mody L. Patient hand colonization with MDROs is associated with environmental contamination in post-acute care. Infect Control Hosp Epidemiol 2017;38: 1110-1113.

6. Jain R, Kralovic SM, Evans ME, et al. Veterans Affairs initiative to prevent methicillin-resistant Staphylococcus aureus infections. N Engl J Med 2011;364:1419-1430.

7. Evans ME, Kralovic SM, Simbartl LA, et al. Nationwide reduction of heatlh care-associated methicillin-resistant Staphylococcus aureus infections in Veterans Affairs long-term care facilities. Am J Infect Control 2014;42:60-62.

8. Hill JN, Hogan TP, Cameron KA, et al. Perceptions of methicillin-resistant Staphylococcus aureus and hand hygiene provider training and patient education: results of a mixed method study of health care providers in Department of Veterans Affairs spinal cord injury and disorder units. Am J Infect Control 2014;42:834-840.

9. Balbale S, Hill J, Guihan M, et al. Evaluating implementation of methicillin-resistant Staphylococcus aureus (MRSA) prevention guidelines in spinal cord injury centers using the PARIHS framework: a mixed-methods study. Implement Sci 2015;10:130.

10. Hill JN, Evans CT, Cameron KA, et al. Patient and provider perspectives on methicillin-resistant Staphylococcus aureus: a qualitative assessment of knowledge, beliefs, and behavior. J Spinal Cord Med 2013;36:82-90. 